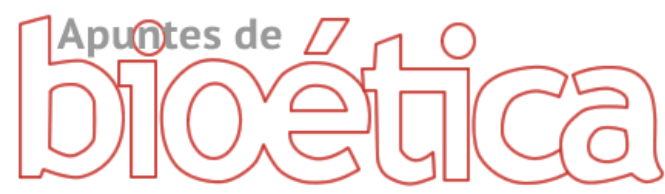

https://doi.org/10.35383/apuntes.v1i1.183

\title{
Directrices para la implementación de un programa de formación en Bioética Personalista dirigido a docentes universitarios
}

\author{
Magna Guzmán Avalos ${ }^{1}$
}

INFORMACIÓN DEL ARTÍCULO RESUMEN

Recibido el 23 de setiembre de 2018

Aceptado el 20 de noviembre de 2018

Palabras claves:

Directrices

Programa de formación

Bioética personalista.
En la actualidad, la educación universitaria exige que el docente tenga una formación integral, lo cual implica disponer de profesores con cualidades positivas que no solo transmitan conocimientos sobre el aspecto académico, el desarrollo científico-tecnológico o el de responsabilidad social, sino que estos, además de su perfeccionamiento profesional, deben poseer vocación de servicio, así como también una sólida formación moral, ética y bioética, capaz de transmitir principios y valores; es decir, ser ejemplo vivo de lo que dicen, considerando al estudiante como una persona en todo su contexto. En este artículo se ha identificado las directrices para la implementación de un programa de formación en Bioética personalista dirigido a docentes universitarios, para lo cual se ha definido la situación actual de la formación docente universitaria en bioética en Perú, así como también se explica los contenidos bioéticos que deben ser considerados en la formación de los docentes universitarios, teniendo como eje transversal de sus ejes temáticos a la Bioética personalista. Las conclusiones a la que se llega es que, La situación actual del docente universitario requiere de una rigurosa formación integral, que incluya a la bioética personalista por el rol de formador que desempeña, impulsando su progresiva implementación en los planes educativos universitarios, a nivel de pregrado y postgrado. Las directrices a implementar son: persona humana, dignidad humana, integridad de la persona y persona íntegra, valor de la vida humana en todas sus etapas, valor de la sexualidad humana, la libertad y el respeto, sociabilidad y socialización.

1 Bachiller en Farmacia y Bioquímica, Maestra en Farmacia Clínica y Bioquímica, Doctora en Gestión y Ciencias de la Educación. Actualmente Directora del Departamento Académico de Propedéutica de la Facultad de Ciencias Médicas. Email: magua_av18@hotmail.com. ORCID: https://orcid.org/0000-0002-4038-3507 


\section{Guidelines for the implementation of a training program in Personalized Bioethics addressed to university teachers}

\section{ABSTRACT}

Keywords:

Guidelines

Training program

Personalistic bioethics
Currently university education requires that the teacher has a comprehensive education, which implies having teachers with positive qualities that not only transmit knowledge on the academic aspect, scientific-technological development or social responsibility, but these, in addition to Their professional development must have a vocation for service, as well as a solid moral, ethical and bioethical training, capable of transmitting principles and values, that is, being a living example of what they say, considering the student as a person in all its context. In this article we have identified the guidelines for the implementation of a personalist bioethics training program aimed at university professors, for which the current situation of university teacher training in bioethics in Peru has been defined, as well as the contents bioethics that should be considered in the training of university professors, having as a transversal axis of their contents the personalist bioethics. The conclusions reached is that, the current situation of the university teacher requires a rigorous comprehensive training, including personalist bioethics for the role of trainer that performs, promoting its progressive implementation in university education plans, at the level of undergraduate and postgraduate. The guidelines to be implemented are: human person, human dignity, integrity of the person and person integrity, value of human life in all its stages, value of human sexuality, freedom and respect, sociability and socialization.

\section{Introducción}

El surgimiento de la Bioética se da partir de 1971, tras la publicación de la obra "Bioethics: Bridge to the future", de Van Rensselaer Potter (1911-2001), “Quien escribió una extensa proposición a manera de tribuna para desplegar esta nueva asignatura, y hasta ahora se toma como punto de referencia para discernimiento de toda acción humana y contribuye al aprendizaje en nuestra sociedad" (GARZA, 2011, p.42). En este contexto, Cantu (2014) afirma:

Las instituciones de educación superior se constituyen, actualmente a nivel mundial, en las instancias de fortalecimiento de los principios bioéticos, esto permitiría además fomentar el desarrollo humano. Al crear espacios permanentes de diálogo, emancipación y formación de profesionales íntegros, se logrará un mundo más humanizado y sustentable, que verdaderamente persuada y fomente el avance de la sociedad hacia contextos situacionales de progreso y apoyo solidario. (p.30)

Asimismo, es necesario enfatizar que, en la actualidad, la docencia universitaria se enfrenta a un panorama heterogéneo de educandos, con diferentes edades, tradiciones, culturas $y$ situaciones políticas. Sumándose definitivamente la presión social procedente del mercantilismo de apertura comercial y de la globalización, que 
viene siendo influenciado por el avance vertiginoso de la ciencia, la tecnología y de la comunicación, conllevando a otra forma de ser del futuro profesional.

Ante esta realidad Cubas, León y Ñique (2018) refieren:

Que el docente universitario debe tener ciertas características que le ayuden a ser modelo a imitar, no solo por su sapiencia científica, sino, sobre todo, por su vocación como persona llamada a servir a los demás, para ello es importante la inclusión en la formación docente en Ética y Bioética en los planes de estudio universitario.

Por consiguiente, existe una preocupación especial en las diferentes escuelas y facultades, incluso en las entidades acreditadoras de educación, para que las instituciones universitarias cumplan con la formación de sus estudiantes en bioética y en humanismo. ( $p$. 47)

Al respecto, es conveniente mencionar que no se han encontrado fuentes bibliográficas que reporten investigaciones sobre la incorporación de la Ética y Bioética en el currículo de las diferentes carreras profesionales que no son de Ciencias de la Salud de las universidades del Perú.

Sin embargo, Cárdenas y Sogi (2013) analizaron que:

De 25 sílabos de cursos de Ética correspondientes a 22 escuelas de medicina peruanas -13 públicas y 9 privadas, solo tres escuelas contaban con dos cursos de Ética siendo las de la Universidad Nacional Mayor de San Marcos, la Universidad Nacional de Piura y la Universidad Peruana los Andes. (p.110)

Analizando la bioética en el Perú podemos observar que:
Los créditos de cursos de ética-bioética en los currículos de las facultades de Medicina del Perú, de lo cual corresponde a la Universidad Católica Santo Toribio de Mogrovejo el 7,59\% de créditos, la Universidad César Vallejo el $7,50 \%$ de créditos, la Universidad Peruana Cayetano Heredia $7,00 \%$ de créditos, la Universidad Nacional Mayor de San Marcos $6,54 \%$ de créditos, la Universidad Nacional de la Amazonía Peruana $6,30 \%$ de créditos, Universidad Nacional José Faustino Sánchez Carrión 5,57\% de créditos, la Universidad Nacional Federico Villarreal 5,19\% de créditos, la Universidad Ricardo Palma 5,10\% de créditos, la Universidad San Martín de Porres $4,06 \%$ de créditos, la Universidad Nacional San Antonio Abad 3,52\% de créditos, la Universidad Católica Santa María 3,09\% de créditos, la Universidad San Luis Gonzaga 3,00\% de créditos, la Universidad Nacional Pedro Ruiz Gallo $2,87 \%$ de créditos y la Universidad Nacional San Agustín 2,02\% de créditos. (Cubas, et al., 2018, p.48)

En tal sentido, el presente artículo pretende responder a la interrogante surgida sobre: ¿Cuáles serán las directrices para la implementación de un programa de formación en Bioética personalista dirigido a docentes universitarios?

Para ello proponemos como objetivo general: identificar las directrices para la implementación de un programa de formación en bioética personalista dirigido a docentes universitarios $\mathrm{y}$, como objetivos específicos: definir la situación actual de la formación docente universitaria en Bioética en Perú. Así como explicar los contenidos bioéticos que deben ser considerados en la formación de los docentes universitarios teniendo como eje transversal de sus contenidos a la bioética personalista. 
La importancia de la investigación se centró en dar respuestas a la interrogante planteada, determinando la necesidad de formular directrices en el campo educativo a través de un programa de formación en Bioética personalista dirigida a docentes universitarios centrado en la persona y en la dignidad humana.

\section{Metodología}

La investigación es de paradigma teórico, de enfoque cualitativo y de tipo documental, con perspectiva integral centrada en la persona, en especial del docente, como sujeto de investigación. Dentro del abordaje metodológico se utilizó la técnica de la observación documental y como instrumentos se emplearon fichas (bibliográficas y de resumen) que han permitido concretar la información recabada, trabajándose también con notas bibliográficas, textuales, de resumen y de síntesis, las cuales permitieron recoger, almacenar, organizar y presentar la información extraída de las fuentes como libros, revistas, entre otras. Finalmente, los resultados de la búsqueda electrónica en internet fueron diversos y de calidad garantizándose el valor científico. El procedimiento se realizó mediante la recopilación del material bibliográfico, que permitió establecer los fundamentos para el desarrollo del objeto de investigación.

\section{Resultados, análisis y discusión}

La presente investigación se centra en la realidad actual de la formación del docente universitario y en la necesidad de incorporar a la Bioética en su formación.

\section{Realidad de la formación del docente universitario en el Perú}

En la actualidad, la universidad peruana se encuentra inmersa en un proceso de cambio con la aplicación de la nueva Ley Universitaria $\mathrm{N}^{\circ}$ 30220 desde el año 2014, que incluye dentro de los deberes del docente: "ejercer la docencia con rigurosidad académica, respeto a la propiedad intelectual, ética profesional, independencia y apertura conceptual e ideológica" (p.39). Sin embargo, existe la imperiosa necesidad de disponer con un docente formado en Ética y en Bioética, para ser referente de los estudiantes que forma. En relación a la ética profesional del docente universitario Orna, (2016) sostiene: "Que es aquel que utiliza el comunitarismo corriente ética y política que afirma la comunidad y su tradición como espacios en los que se da la vida buena: realización de la vida humana" (p.293). En consecuencia, el autor busca sustentar la relación entre fines, normas y virtudes. Los fines son los ideales que orientan la actividad. Las normas nos ayudan a encaminarnos hacia ese fin, son como el mapa que nos lleva a donde queremos. Las virtudes son las disposiciones necesarias para emprender la caminata. Asimismo, respecto a la formación del docente en el Perú, Orna, en su investigación Ética del Docente Universitario, realizada en la Universidad Nacional Mayor de San Marcos, reporta como conclusión que:

El rol y responsabilidad de todo profesional universitario con la sociedad no se limita solo a trasmitir conocimientos, además debe brindar los fundamentos intelectuales de la vida moral y el desarrollo del sentido de aquellas realidades que son espirituales por naturaleza, como la verdad y la belleza. (p.294)

Desde otra perspectiva, Sovero (2012) en su tesis sobre la formación docente universitario, desarrollada en la Universidad Católica Santo 
Toribio de Mogrovejo concluye que: "Los docentes de las diferentes asignaturas requieren retroalimentación en la formación de Ética y Bioética como educación continua y actualizada para que puedan abordar los temas en forma concreta para saberlos transferir en las diferentes temáticas" (p.60).

Otra debilidad en la formación del docente en el Perú son los valores. Al respecto, Hodelín y Fuentes (2014) sostienen que: "Las universidades deben centrarse en la formación de un profesional integral que unido a una sólida preparación científica y técnica, exhiba su compromiso social y formación humanística, lo cual se relaciona directamente con los valores éticos" (p.2). La enseñanza en valores requiere, por parte del docente, lograr el desarrollo de valores en sus estudiantes; en tal sentido, respecto al desempeño docente en la práctica de valores, Benites, J., (2017) en su tesis desarrollada en la Universidad Privada Norbert Wiener, concluye que:

La práctica de valores en el desempeño docente y el nivel de percepción de los estudiantes, presentan una valoración porcentual de 0,34 determinando un grado significativo de relación del $88,67 \%$, corroborando la siguiente hipótesis: la práctica de valores en la enseñanza está en relación directa con el nivel de percepción de los estudiantes. (p.72)

Como se puede apreciar la formación ética y bioética en los docentes universitarios es escasa, con lo cual urge mecanismos que ayuden a solucionar esta carencia en bien de la comunidad universitaria y de la sociedad en su conjunto.

\section{La Bioética y el rol del docente universitario en el Perú.}

Otro acápite a analizar en este artículo es la de analizar cómo se ha desarrollado la bioética en el Perú. Al respecto Llanos (2012) sostiene que: "La Bioética no ha adquirido todavía identidad propia y refiere que esta disciplina es algo más que los problemas éticos de la práctica médica" (p.1). Referente al rol del docente, el estudio más reciente es el de Gutiérrez (2015) quien en su tesis concluye que: "La Universidad Nacional Mayor de San Marcos cuenta con un marco ideológico y conceptual que le da soporte a sus planteamientos sobre Responsabilidad Social Universitaria, lo cual constituye un marco favorable para la incorporación de la Bioética" (p.8).

La Bioética constituye un papel importante en el docente universitario quien requiere del conocimiento de esta para su formación y también para inculcar lo positivo a los nuevos profesionales. Las investigaciones dan cuenta de avances en este campo de la vida, de las dificultades para determinar el carácter ético de la ciencia y del nacimiento en tal sentido "Bioética como mediadora entre el conocimiento científico-técnico y la educación superior; es decir, como garante de una formación equilibrada de los profesionales" (Salas, 2015, p.112).

Al analizar la Bioética y el rol del docente universitario, aseveramos que existe la necesidad de incluirla como parte de su formación para el ejercicio de la docencia universitaria. Al respecto, Fernández (2012) refiere que:

El profesor sirve de modelo profesional a reproducir por el alumno, dirige y corrige su interacción con el objeto y la búsqueda y asimilación de la teoría pertinente, así como también orienta y direcciona su aprendizaje, 
fundamentalmente hacia la formación de valores. Por ello se resalta que profesores, escuela, familia y sociedad constituyen referentes para que el estudiante universitario se forme y se desarrolle en su integridad. (p.362)

De lo dicho anteriormente, resulta necesaria apostar por la formación bioética por parte de los docentes universitarios.

\section{Incorporación de la Bioética personalista}

La investigación tiene como eje central la incorporación de la Bioética personalista en la educación universitaria, para ello se considera la propuesta de unas directrices en la formación docente; en este sentido Yacarini (2013), considera que:

Los planteamientos bioéticos brindan un aspecto novedoso favoreciendo la reflexión y el diálogo de los dilemas bioéticos vistos desde una perspectiva personalista lo cual permite una promoción y la defensa de la vida desde el momento mismo de la concepción hasta la muerte natural, respetando la dignidad humana y la recta conciencia moral. (p.10)

Cuando se estudia la bioética personlista, necesariamente se debe hacer referencia a sus principios propuestos por Sgreccia (2014), porque presentan una visión universalista y ontológica de la dignidad humana. A continuación se hace una breve referencia de los mismos:

a) El principio de defensa de la vida física destaca que la vida corpórea es el valor fundamental de la persona porque esta no puede existir si no es en un cuerpo como co-esencial a la persona; es decir, como base única y necesaria para su existencia en el tiempo y en el espacio. Tampoco la libertad puede darse sin la vida física: para ser libre y tener derechos humanos es necesario ser viviente. No se puede ser libre ni defender los derechos básicos si no tenemos la vida. La vida llega anteriormente a la libertad. Por lo tanto, cuando la libertad suprime la vida es una libertad que se suprime a sí misma $y$, en consecuencia, elimina la base de los derechos humanos. Solo el bien total y espiritual de la persona está por encima del valor de la vida física.

b) El principio de totalidad acentúa que la persona humana con el organismo corpóreo constituye una totalidad y el organismo mismo es una totalidad. Para Sgreccia, la corporeidad humana es un todo unitario que resulta de partes distintas, orgánica y jerárquicamente unificadas entre sí por la existencia única y personal. De aquí se deriva el siguiente principio que va estrechamente unido al anterior.

c) El principio terapéutico, por el cual es lícito intervenir en una parte del cuerpo cuando no hay otra forma para sanar o salvar su totalidad.

d) El principio de libertad y responsabilidad engloba el concepto de que la persona es libre, pero es libre para conseguir el bien de sí misma y el bien de las otras personas y de todo el mundo, pues el mundo ha sido confiado a la responsabilidad humana. No puede ejercitarse la libertad sin ejercerse la responsabilidad. Se debe procurar una Bioética de la responsabilidad frente a las otras personas, frente a sí misma y, ante todo, a la propia vida, a la vida de los 
otros hombres y la de los otros seres vivos. No obstante, como se ha dicho en el primer principio, el derecho a la defensa de la vida tiene prioridad sobre el derecho a la libertad; es decir, para ser libres es imprescindible estar vivos porque la vida es condición necesaria para el ejercicio de la libertad.

e) El principio de la sociabilidad y subsidiariedad pone de relieve que toda persona está obligada a autorrealizarse participando en la realización del bien de sus semejantes $y$, dado que la vida humana es un bien personal y también social, cada persona debe comprometerse a proteger la vida como un patrimonio de la sociedad y no solo de cada individuo personal. Asimismo, la subsidiariedad es un principio que completa al anterior diciendo que la sociedad tiene la doble obligación de asistir o ayudar más allí donde las necesidades son más graves y urgentes, sin suplantar o sustituir las iniciativas libres de los ciudadanos, bien sea individualmente o de forma asociada. Este principio implica la práctica de la solidaridad. (págs. 218-228)

Otro punto a rescatar en el estudio de la bioética personalista es el estudio a través del Ilamado Método triangular (Sgreccia, 2014), que consiste en un examen que tiene tres puntos de enlace, divido de la siguiente forma: El vértice $A$, es la exposición de un hecho biomédico; este hecho debe ser comprobado científicamente en su consistencia y exactitud. En otras palabras, constituye el análisis de los datos científicos. El vértice $B$ es la profundización del significado antropológico del hecho biomédico; es decir, analiza los valores que están en juego en relación con la vida. Según Pessina, citado por Screccia (2014) "a partir de este examen se podrá determinar qué valores hay que defender y cómo se deben regular la acción y los agentes en el plano individual y social", el examen de los valores se debe basar en el diálogo profesor alumno, lo cual debe ser intrincado llevar a la práctica debido a la estructura de los centros de enseñanza, con gran cantidad de alumnos por profesor. El vértice $C$ refiere la filosofía del hombre en su conjunto; es decir que, en la solución a los problemas éticos se debe buscar la relación de los conceptos con los valores fundamentales de la persona humana, este es el momento de determinación de las exigencias morales del obrar para el caso concreto. Mediante este método, el protagonista de su propia formación es el estudiante, quien debe realizar la tarea de buscar, preparar y asimilar los conocimientos. La tarea del profesor se limita a guiarle en la consecución de su labor.

En consecuencia, el método triangular considera primero atender a la persona como un todo, en una mirada integral que abarque no solo el cuerpo, sino también la psique y el espíritu. En segundo lugar, contemplar a la persona como un ser estructuralmente abierto a los demás (lo que resulta muy relevante a la hora de calibrar las consecuencias sobre terceros de determinados cursos de acción). Por último, es preciso considerar a la persona, a lo largo de toda su existencia, siempre como un fin en sí misma y nunca un medio para la obtención de fines ajenos a ella. (Alberth, 2018, p.7)

De allí que López (2012), establezca que la enseñanza y aprendizaje de la Bioética personalista en el ámbito universitario se debe analizar el escenario en el que se desarrolla la Bioética en los currículos universitarios, y la disyuntiva entre las dos orientaciones, (Bioética personalista o Bioéticas personalistas) lo cual se debe tener en cuenta en la formación de la docencia de la Bioética personalista, bien porque 
generan problemas o discrepancias, o bien porque no tenerlas suficientemente clarificadas dificulta la enseñanza y el aprendizaje. (p. 83)

\section{Propuesta de las directrices para la implementación un programa de formación en bioética personalista dirigido a docentes universitarios:}

Luego de lo expuesto, se puede afirmar que existe la necesidad de formular algunas directrices para implementar un programa de formación en Bioética personalista dirigido a docentes universitarios, para ello nos aventuramos a proponer las siguientes:

\section{La persona humana.}

La primera directriz está centrada en la persona, con ello se busca dar importancia a la responsabilidad de la labor docente que es la de fomentar y coadyuvar al crecimiento de la condición humana de sus estudiantes, contribuyendo a su desarrollo y así convertirlos en ciudadanos dignos de estima que desempeñen un papel positivo en la comunidad.

El docente universitario debe formar a los estudiantes enseñándoles a actuar sobre lo más propio y distintivo de cada ser humano, en su singularidad, en su dignidad humana, su autoestima y autorrealización; lo cual contribuirá en los estudiantes a desarrollar una actitud de reflexión, capacidad de pensamiento crítico, cooperativo, humanista, flexible, creativo y una formación sólida ética y estética ante situaciones sociales en diferentes contextos; conocer, querer, elegir, sentir, expresarse, comportarse, relacionarse con los demás y responsabilizarse por su propio actuar; también cuestionarse el lugar de la ciencia y la tecnología en el progreso humano. Lo vertido significa formar profesionales con una ética profesional que busca desarrollar armónica y coherentemente al ser humano, con el único fin de lograr su realización plena dentro de la sociedad. En la medida en que dicha formación sea reconocida, comenzará un proceso de búsqueda de una teoría que justifique lo que se hace; de allí que surgen las motivaciones, se amplían los intereses y se profundizan los sentimientos, convirtiéndose por ello en alternativa viable para la formación integral del profesional. (Loret de Mola, 2015)

\section{La dignidad humana.}

La segunda directriz está referida a la dignidad humana, esta se plantea desde la perspectiva de que el docente desempeña un rol preponderante en la formación de los estudiantes universitarios, es por ello que el docente debe poseer cualidades dirigidas al respeto de la dignidad de la persona, reflejada en los estudiantes.

\section{Integridad de la persona y persona íntegra.}

Consideramos importante este aspecto porque el docente universitario debe trasmitir que la integridad es la cualidad de la persona a ser honesto consigo mismo y con los demás; es decir, que la persona debe aprender a vivir una vida que esté de acuerdo con sus valores personales. Se aspira a estudiantes integrales que practiquen la honradez, bondad, modestia, solidaridad, responsabilidad, amistad, fortaleza, respeto, prudencia, fidelidad, pertenencia, honestidad, tolerancia, ética y justicia. Se consideran estos valores sin conferirle ningún grado de prioridad. Ello es importante porque el estudiante universitario los aplicará en las diversas circunstancias en las que se encuentre. 
Consecuentemente, la integralidad permitirá que la vida social de dichos estudiantes alcance los más altos elogios que una sociedad puede brindar" (Gonzales, 2018).

\section{El valor de la vida humana en todas sus etapas.}

La cuarta directriz es importante porque permite enseñar en forma adecuada $y$ responsable el valor de la vida humana en todas sus etapas, ya que esta no es un mero hecho o modalidad de existencia natural, social o racional, sino que encierra un valor en cada etapa del desarrollo de la persona.

\section{El valor de la sexualidad humana, la libertad y el respeto.}

La quinta directriz que se propone, es importante porque se considera fundamental el aprendizaje del estudiante sobre la amplitud y el sentido de la sexualidad humana, así como de los actos contrarios a esta virtud, en éste sentido, la universidad se siente comprometida socialmente con la formación integral del estudiante, por eso se requiere la existencia de planes curriculares donde exista asignaturas que permita el desarrollo exitoso de la educación de la sexualidad, en particular hacia elementos específicos como la identidad sexual, el sexos, orientación sexual y las relaciones de pareja, entre otras expresiones de afectos, además de la castidad. Para ello es necesario preparar a los docentes con conocimientos, habilidades y actitudes de apertura que permita romper concepción heterosexista, con el compromiso de trasmitir a sus estudiantes una forma de pensar, sentir y conducta centradas en valores. (Martínez et al. 2012)

\section{Sociabilidad y Socialización.}

La sexta directriz propuesta es importante porque el docente en el proceso de enseñanzaaprendizaje debe fomentar la tendencia a vivir en sociedad $y$ en comunidad y en este proceso debe internalizar el respeto a las normas $y$ valores, al entorno social, teniendo como fin la integración del individuo en la sociedad, donde le toque desempeñar. El estudiante universitario es el sujeto que actúa en la sociedad, construye y modifica su cultura, transforma el espacio y sus vínculos con los otros sujetos según el contexto histórico concreto y los sistemas de valores referenciales, institucionalizados o no. En tal sentido, los docentes deben enseñar a sus estudiantes las cualidades humanas sin olvidar su vínculo con la sociabilidad y la relación universidad-sociedad (Negret, 2015).

\section{Conclusiones}

La situación actual del docente universitario requiere de una rigurosa formación integral que incluya a la Bioética personalista por el rol de formador que desempeña, impulsando su implementación en los planes de estudios universitarios, de pregrado y postgrado.

En el contenido bioético para la formación del docente universitario se debe considerar principalmente como eje transversal de sus contenidos a la Bioética personalista, así como también la Educación en valores, lo que se define como un proceso humanizador, individual, social, vertical y horizontal a lo largo de la vida de las personas.

Las directrices que se han definido para la implementación de un programa de formación 
en bioética personalista dirigido a docentes universitarios son: La persona Humana. La dignidad Humana. Integridad de la persona y persona íntegra. El valor de la vida humana en todas sus etapas. El valor de la sexualidad humana, la libertad y el respeto. Sociabilidad y Socialización.

\section{Recomendaciones}

Ante la realidad actual en el desempeño de sus funciones de los docentes universitarios; es de vital importancia que el sistema de la universidad peruana implemente las directrices en bioética personalista a través de un programa de formación dirigido a docentes universitarios.

La Superintendencia Nacional de Educación Superior (SUNEDU) debería disponer la incorporación de la bioética personalista en los planes de estudio universitarios a nivel de pregrado y postgrado, con la finalidad de garantizar la formación integral de los estudiantes.

\section{Bibliografía}

Albert, M. (2018). ¿Bioética sin metafísica? Antropología y deliberación moral en el Bioethics Core Curriculum de la UNESCO". Madrid, España.

Cantu, C., (2014). La bioética en las instituciones de educación superior en el contexto de la sustentabilidad. Nuevo León, México

Cárdenas M. y Sogi C. (2013). Enseñanza de la ética en las escuelas de medicina peruanas: un estudio de sílabos. An. Fac. Med. v.74 n.2 Lima abr./jun.
Cubas, F., León, F. y Ñique, C. (2018). Educar en Bioética: Retos para el profesor universitario de ciencias de la salud. Rev. Med. Hered. 29:46-51.

Congreso de la República, (2014). Ley Universitaria $N^{\circ} 30220$, Lima, Diario Oficial el Peruano Normas Legales. p 527223

Garza, V. (2011). Bioética y educación ambiental. CULC y T: Cultura Científica y Tecnológica, 8 (42):

Gutiérrez, A. (2015). Conocimiento y Aplicabilidad de la Bioética: UNMSM - Perú y Culagos/Guadalajara - México. Revista de la Facultad de Ciencias Contables Vol. $23 \mathrm{~N}^{\circ} 43$ pp. 41-49. UNMSM, Lima - Perú.

Hernández, R. (2014). Metodología de la Investigación, $6^{\mathrm{a}}$. Edi. Prentice Hall, México.

López, R. (2013). Ética de la Docencia Universitaria. La Dimensión Social en la Cultura Profesional del Profesorado. Universidad de Autónoma de Sinaloa. Rev. EDETANIA.

Llanos, R. (2012). Historia de la bioética en el Perú en el siglo $X X$, Pontificia Universidad Católica del Perú. Lima, Perú.

Salas, F. (2015). La bioética y el rol del docente universitario en la formación del enfermero. Universidad el Bosque. Revista Colombiana de Bioética. Vol. $10 \mathrm{~N}^{\circ} 1$

Sgreccia, E. (2014). Manual de Bioética I: Fundamentos de ética y bioética, $2^{\mathrm{a}}$ impresión, Madrid, Biblioteca de Autores Cristianos. $218-228$ 
Sovero, E. (2012). La Enseñanza de la Ética y Bioética como eje transversal. Escuela de Enfermería. (Tesis de Maestría). Universidad Católica Santo Toribio de Mogrovejo. Chiclayo, Perú.

Tapia, A., (2016). Formación Didáctica de Docentes Universitarios Haciendo Uso de la Modalidad Virtual. (Tesis de Materia). Pontificia Universidad Católica del Perú. Lima, Perú.

Yacarini, A. (2013) Bioética, Formación y Educación. (Tesis de Maestría). Universidad Católica Santo Toribio de Mogrovejo. Chiclayo, Perú.

\section{Páginas electrónicas}

Alcázar, J. (5 de febrero del 2018). Educar en y para la libertad. EDUCREA. Recuperado de: https://educrea.cl/educar-en-y-para-la-libertad/

Comstock, G. (5 de febrero del 2018). Ética de la investigación: una guía filosófica para la conducción responsable de la investigación. Scopus. Recuperado de: www.scopus.com/record/display.uri? eid=2s2. 085016163119\&origin =inward\&txGid =e83ce a7a2ad62ab05bda068a7a625d78

Chapa, P. y Martínez, T. (6 de febrero del 2018). Valores Universitarios en los Jóvenes Estudiantes de la Facultad de Ciencias Políticas y Administración Pública. RIDE. Recuperado de: https://www.ride.org.mx/index.php/RIDE/articl e/viewFile/127/563

Domínguez, L. (6 de febrero del 2018). La formación de valores en jóvenes universitarios. UH. N.278 La Habana
Recuperado

de: http://scielo.sld.cu/scielo.php?script=sci_artte xt\&pid =S0253-92762014000200007

Fernández, J. (5 de febrero del 2018). Educación médica: el objeto, el sujeto desdoblado y el contexto. Scielo. Recuperado de: http://scielo.sld.cu/scielo.php?script=sci_artte xt\&pid $=$ S086421412000300001\&lng =es

González, R. y Cardentey, J. (6 de febrero del 2018). Educación en valores de estudiantes universitarios. Humanidad Médica. Recuperado de: http://scielo.sld.cu/scielo.php?script $=$ sci_artte xt\&pid=S1727-81202016000100011

Hodelín, R, Fuentes, D. (5de febrero del 2018). La solidaridad más allá de la justicia: aproximaciones al escenario cubano. MEDISAN. Recuperado de: http://scielo.sld.cu/scielo.php?script=sci_artte xt\&pid=S1029301920120012\&lng=es

Loret De Mola, E., Pino, D. y Nordelo, J. (6 de febrero del 2018]. La formación humanística en las carreras universitarias cubanas. Humanidad Médica. Recuperado de: http://scielo.sld.cu/scielo.php?script=sci_artte xt\&pid $=$ S1727-81202015000100002

Martínez De León, B., Puig, A., Ulloa, I. y Serret, J. (6 de febrero del 2018). Estrategia para la educación de la sexualidad en estudiantes de la carrera de Psicología. Red de Revistas Científicas de América Latina, el Caribe, España y Portugal. Recuperado de: http://www.redalyc.org/pdf/4773/4773489480 06.pdf

Negret, J., (6 de febrero del 2018]. Formación ciudadana, cultura física y deporte: estrategia para una formación de calidad. Scielo Cuba 
Recuperado

de:

Recuperado

de:

http://www.efdeportes.com/efd199/la-

https://www.uaeh.edu.mx/scige/boletin/huejut

formacion-ciudadana-a-traves-de-una-gloria-

deportiva.htm

la/n9/e2.html

Orna, O. (6 de febrero del 2018). Ética del

UNIVERSIA Perú. (5 de febrero del 2018). La Docente Universitario. Humanidades Médicas. Recuperado de: revistas.urp.edu.pe/index.php/Inkarri/article/do wnload/90/80/

RODRIGUEZ, H. (6 de febrero 2018). Importancia de la formación de los docentes en las instituciones educativas. Universidad del Estado de Hidalgo, Ciencia Huasteca deficiente formación de los docentes es el principal problema de la educación básica en Perú. UNIVERSIA Perú. Recuperado de: http://noticias.universia.edu.pe/educacion/noti cia/2017/10/11/1156127/deficiente-formaciondocentes-principal-problema-educacionbasica-peru.html 